CHRONIQUE DE LA RECHERCHE

\title{
LE LIT DE JUSTICE ET LA MONARCHIE FRANÇAISE Alain BOUREAU
}

Depuis une quinzaine d'années, la connaissance du rôle du lit de justice dans le fonctionnement de la monarchie française d'Ancien Régime a été complètement renouvelée ${ }^{1}$. On le sait, le lit de justice, du moins tel qu'il était compris en sa phase finale, au XviIr ${ }^{\mathrm{e}}$ siècle, se définissait comme une séance solennelle du parlement de Paris où le roi imposait l'enregistrement d'édits, en s'épargnant ainsi des négociations ou des échecs. L'expression elle-même évoquait le mobilier cérémoniel qui exprimait et exhibait cette toute-puissance législative et judiciaire du souverain, en différenciant formellement le lit de justice des séances ordinaires, qu'elles aient lieu ou non en présence du roi.

$\mathrm{Ce}$ renouveau tient certes au progrès des recherches empiriques, mais aussi à des changements de cadre historiographique; en effet, on peut distinguer trois phases successives dans l'étude du lit de justice.

Dans un premier temps, le lit de justice, comme d'autres formes cérémonielles de la vie politique d'Ancien Régime, a été considéré comme un objet historique secondaire, cantonné dans le genre de l'« histoire des institutions ", telle que la pratiquaient des savants comme François Olivier-Martin ou Roger Doucet; à l'intérieur de ce genre, qui tendait à faire des rouages institutionnels des invariants de l'histoire politique, les formes cérémonielles ne recevaient qu'une modeste attention. Par ailleurs, les nouvelles formes d'histoire, à partir des années 1930, ont contribué à rejeter l'institution et plus encore la cérémonie dans le magasin des accessoires futiles qui décoraient une scène à laquelle l'histoire sociale devait donner un sens plus réel. Qu'on se rappelle la fameuse polémique entre Lucien Febvre et Henri Jassemin en $1934^{2}$. Jassemin avait écrit une histoire de la Chambre des comptes qui la traitait un

1. Sarah HANLEY, The Lit de justice of the kings of France. Constitutional ideology in legend, ritual and discurse, Princeton, Princeton University Press, 1983, trad. franç. André ChARPENTIER, Le "Lit de justice" des rois de France. L'idéologie constitutionnelle dans la légende, le rituel et le discours, Paris, Aubier, 1991. Mack P. HolT, « The king in Parliament. The problem of the lit de justice in sixteenth-century France », The Historical Journal, 31, 1988, p. 507-523. Elizabeth A. R. Brown, Richard C. FamiglietTi, The Lit de justice. Semantics, cerimonial and the parliament of Paris. 1300-1600. Sigmaringen, Jan Thorbecke Verlag, 1994.

2. Voir Lucien Febvre, «Comptabilité et Chambre des comptes», Annales d'histoire économique et sociale, t. 6, 26, 1934, p. 76-81.

Revue de synthèse : $4^{e}$ S. $\mathrm{N}^{\mathrm{os}}$ 2-3, avril-septembre 1997, p. 285-291. 
peu comme notre actuelle Cour des comptes; Febvre répondait avec véhémence en déniant toute existence réelle à l'institution, simple décor sans profondeur. Entre l'anhistoricité et l'historicisme, la cérémonie politique a longtemps échappé à l'analyse.

En un deuxième temps, avec la parution du travail de Sarah Hanley, en 1983, le lit de justice émerge comme objet historique complexe et significatif, profondément inscrit dans l'histoire. Cet ouvrage doit se saisir au sein d'une tendance plus ancienne : à partir des années 1950, aux États-Unis, se construisait une nouvelle recherche sur les grandes cérémonies de la monarchie française d'Ancien Régime. L'initiateur en avait été Ralph Giesey, dans sa thèse dirigée par Ernst Kantorowicz. (mais achevée avant la publication des Deux Corps du roi), qui portait sur les funérailles royales; puis avaient suivi les travaux de Richard Jackson sur le sacre et le couronnement et de Lawrence Bryant sur les entrées ${ }^{3}$. Au-delà de la thématique commune, ces auteurs partagent un même souci de montrer que ces rites politiques se comprennent comme illustration d'une «idéologie constitutionnelle " de la monarchie, dont la formulation verbale n'intervient qu'après l'élaboration cérémonielle. Sarah Hanley, elle-même élève de Ralph Giesey, a donc achevé cette exploration avec sa recherche sur le lit de justice.

Enfin, avec une troisième phase, marquée notamment par un ouvrage d'Elizabeth A. R. Brown et de Richard C. Famiglietti publié en 1994, le moment semble venu d'une lecture plus contextuelle et plus ouverte du rite politique, plus sensible aux interactions et aux processus. Mais avant de spécifier ces orientations, il convient d'examiner les apports du livre de Sarah Hanley, qui a eu le mérite de montrer que l'institution du lit de justice avait une histoire complexe et variée et donc de le construire en objet historique.

Pour Sarah Hanley, l'expression « lit de justice » apparaît dès le $\mathrm{xIV}^{e}$ siècle, mais jusqu'en 1527, elle ne désigne guère davantage que l'appareil matériel (structure de bois, coussins, tentures) qui entoure la présence solennelle du souverain en son Parlement. La cérémonie ne vise qu'à donner une image majestueuse de la justice française, exercée conjointement par le roi et par le Parlement. Mais tout change au $\mathrm{XvI}^{\mathrm{e}}$ siècle, quand on distingue soigneusement la séance royale, où la présence du roi n'a rien de solennel et qui est consacrée généralement à des questions fiscales et judiciaires, du lit de justice, marqué par un apparat particulier et par la présence des proches, et qui traite de ce que l'auteur appelle des questions constitutionnelles relevant du droit public. La première occurrence du lit « constitutionnel » se situerait en 1527, sous François $1^{\text {er }}$, lorsqu'il s'agit de juger Charles de Bourbon, puis de revenir sur les termes du traité de Madrid. L'enjeu de la cérémonie est de donner publiquement au roi une capacité législative que lui conteste le Parlement. Les présidents du

3. Ralph E. Giesey, The Royal Funeral Ceremony in Renaissance France, Genève, Droz, 1960, trad. franç. Jeannie CARLIER, Le Roi ne meurt jamais. Les funérailles royales au temps de la Renaissance, préf. François FurEt, Paris, Flammarion, 1987. Richard A. JACKSON, Vive le Roi! A history of the French coronation ceremony from Charles $V$ to Charles $X$, Chapel Hill, University of North Carolina, 1984, trad. franç. Monique Arav, Vivat Rex. Histoire des sacres et couronnements en France, Strasbourg, Association des publications près les universités de Strasbourg, 1984. Lawrence Bryant, The King and the city in the Parisian royal entry ceremony. Politics, ritual and art in the Renaissance, Genève, Droz, 1986. 
Parlement, en 1527, semblent d'ailleurs refuser de considérer que le lit de justice differe d'une séance royale ordinaire. Le souci d'innovation institutionnelle se marque, à partir des années 1540-1550, par une activité érudite, notamment chez Jean Du Tillet, greffier civil au parlement de Paris de 1530 à 1570, qui construit la légende d'une existence médiévale du lit de justice, signe ancien des prérogatives royales. Le chancelier Michel de L'Hospital, vers 1560, prend le relais de Du Tillet. Après une éclipse sous Henri II et François II, Charles IX revient à la pratique du lit de justice constitutionnel; en 1563, une nouvelle fonction juridique du lit apparât : la cérémonie marque la majorité du roi, son immédiate capacité à dire les lois. À partir de 1610, lorsque le jeune Louis XIII assiste à un lit de justice, au lendemain de l'assassinat de son père Henri IV, Sarah Hanley distingue un deuxième âge du rite politique; sa finalité, de juridique, devient dynastique : le président de Harlay, dans son discours, affirme l'aspect instantané, «biologique» de la succession royale; dès lors, le lit de justice tend à prendre la place institutionnelle du sacre et du couronnement. Une deuxième vague d'érudition monarchiste se manifeste dans les premières décennies du Xvir siècle (La Roche Flavin, les Godefroy, Du Chesne, etc.). Enfin, à partir de Louis XIV et du lit de 1643, la cérémonie prend un tour absolutiste: la monarchie n'y définit plus les principes du droit public, mais se montre en majesté et impose sa volonté au Parlement.

Le livre de Sarah Hanley a le grand mérite de reconstituer l'histoire d'une institution fort méconnue : l'auteur a consulté un grand nombre de sources sur le lit de justice et nous en donne une chronique détaillée. Ses deux chapitres sur le tournant de 1610 , qui examinent le rôle respectif des diverses formes cérémonielles de l'avènement royal sont vraiment remarquables. Cependant, son interprétation des sources me paraît susciter des réserves importantes.

Le pivot de la démonstration, désigné par le sous-titre de l'ouvrage, se trouve dans l'« idéologie constitutionnelle» de la France du xvi siècle : une véritable constitution de la monarchie s'élaborerait d'abord dans les rituels, puis, à la fin du siècle, dans les textes. Cette conception, partagée par Ralph Giesey et les « néo-cérémonialistes » américains, trouve son origine moins chez le mâtre de Giesey, Ernst Kantorowicz, que dans l'ouvrage de William F. Church, Constitutional Ideology in xvith century France ${ }^{4}$ ou dans l'œuvre de Joseph Strayer. Or cette idée, projetée à partir de la situation de la monarchie anglaise de l'époque élisabéthaine ou même de la création de la constitution américaine au XVIII siècle, n'a pas de pertinence dans la France du $\mathrm{xvI}^{e}$ siècle : la distinction entre droit privé et droit public n'y existe guère. La construction essentialiste d'un « pré-droit » constitutionnel, à l'état virtuel, semble donc bien arbitraire. Dès lors, la démonstration du caractère constitutionnel des lits de 1527 devient bien laborieuse; l'auteur doit sans cesse transcrire ou traduire un discours manifeste en une suite de propositions latentes : pour elle, l'accusation de lèse-majesté contre Charles II de Bourbon, coupable d'être passé à l'ennemi, relève, on ne sait trop pourquoi, " de la loi d'inaliénabilité, c'est-à-dire du droit public ${ }^{5}$ ». De même, le discours du président Guillart est présenté comme une manifestation de résistance à l'entreprise du roi uniquement par ses silences et ses

4. Cambridge, MA, Harvard University Press, 1941.

5. S. HANLEY, op. cit. supra n. 1, p. 50. 
omissions. Pour établir la nouveauté de ce pré-droit rituel, en ses diverses phases, Sarah Hanley note des émergences trompeuses : ainsi la métaphore du mariage politique du souverain avec la République se trouverait pour la première fois dans le discours du président Jean de Selve au lit de décembre 1527; or les travaux récents de Robert Descimon ${ }^{6}$ montrent clairement que cette métaphore circule dans le milieu parlementaire au moins depuis le temps de Louis XI. De même, l'innovation «dynastique » dans le discours du président de Harlay lors du lit inaugural de 1610 serait marquée par l'emploi d'expressions qui affirment l'identité «biologique » entre Henri et Louis, « image vive du défunt ». Or cette expression a été employée littéralement par Pierre Du Chastel, évêque de Châlons, dans son oraison funèbre lors des obsèques de François $1^{\text {er }}$, le 23 mai 1547 à Notre-Dame ${ }^{7}$. En remontant encore dans le siècle, on trouverait en 1532, chez Rabelais, la lettre de Gargantua à son fils Pantagruel, dans laquelle le père console ainsi le fils de sa mort future: «Par quoy, ainsi comme en toy demeure l'image de mon corps, si pareillement ne reluysaient les meurs de l'âme, l'on ne te jugerait estre garde et trésor de l'immortalité de nostre nom ${ }^{8}$. " Ces rectifications de détail importent, car elles mettent en cause l'autonomie du lit de justice comme «forum constitutionnel », comme rite; en effet, les affirmations posées au cours du lit relèvent de discours qui pourraient se tenir ailleurs et qui ont leur propre généalogie; l'aspect rituel du lit n'a pas de corrélation nécessaire avec les propos tenus. Mais alors comment ne pas revenir à une idée de la cérémonie politique comme décor, comme monotone gestuelle du pouvoir?

Fort heureusement, l'ouvrage d'Elizabeth Brown et de Richard Famiglietti prend un efficace relais. Ce livre, paru onze ans après celui de Sarah Hanley, semble limiter son objet à un réexamen de l'objet sur une période plus courte (1300-1600 - en fait 1574, car l'analyse s'arrête à la mort de Charles IX), en s'attachant à montrer que l'existence précoce du lit de justice a été indûment négligée par Sarah Hanley. Ce propos critique, qui apparaît surtout dans les notes de bas de page, risque de passer inaperçu, mais, en fait, au-delà de ce réajustement, cette étude propose une véritable alternative dans l'interprétation.

Pour Sarah Hanley, le lit de justice n'a d'existence cohérente (parce que « constitutionnelle ») qu'à partir de 1527, avec une vague esquisse sous le règne de Charles VI; à l'époque médiévale, il désignerait essentiellement l'appareil matériel qui entoure certaines apparitions du roi au Parlement; or Brown et Famiglietti montrent que, depuis une ordonnance de Philippe V, en 1318, qui mentionne pour la première fois la présence d'un « lit » royal au Parlement, même s'il n'est pas encore « de justice ", les formes cérémonielles et politiques de présence du roi au Parlement ne cessent de s'élaborer; tout au long des $x v^{e}$ et $\mathrm{Xv}^{\mathrm{e}}$ siècles, au-delà des notations de vocabulaire, une pompe ample, une présence dense de hauts personnages

6. Robert Descimon, «Les fonctions de la métaphore du mariage politique du roi et de la République, France, $\mathrm{xv}^{\mathrm{e}}$-xvi11 ${ }^{\mathrm{e}}$ siècle », Annales. E.S.C., 6, 1992, p. 1127-1147.

7. Voir Alain Boureau, « Les cérémonies royales françaises entre performance juridique et compétence liturgique », Annales. E.S.C., 6, 1991, p. 1253-1264, et ID., « Ritualité politique et modernité monarchique. Les usages de l'héritage médiéval », in L'État ou le roi. Les fonda-

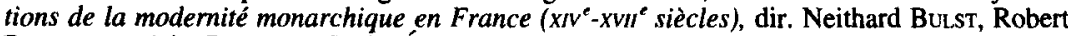
Descimon, Alain Guerreau, Paris, Éd. de la Maison des sciences de l'homme, 1996, p. 9-25.

8. François Rabelais, Pantagruel, chap. vilI. 
associent étroitement le souverain à la fonction de haut justicier, notamment à l'occasion des procès en trahison ou en lèse-majesté. Ainsi, le procès de Robert d'Artois en 1332 inaugure une longue série de cas analogues. Or ce procès au Parlement a fait l'objet de deux représentations picturales contemporaines qui notent la disposition singulière des lieux de justice en cette occasion. C'est dire que ce type d'appropriation souveraine du Parlement préexiste à sa dénomination particulière. À l'époque de Charles $\mathrm{V}$, les séances royales au Parlement sont liées à la proclamation nouvelle de la majesté royale, prise alors non comme un vague attribut, mais comme le fondement juridique de la souveraineté monarchique. Le témoignage de Christine de Pisan, en 1404, mal interprété par Sarah Hanley, est révélateur : pour elle, le roi tient lit de justice « es cas reservez à terminer à lui »; or, on le sait, les « cas réservés » au roi gravitent autour de la notion de lèse-majesté. Le lit de justice médiéval n'est donc nullement une invention de Jean Du Tillet, mais une forme justicière de l'affirmation de la souveraineté royale.

Les découvertes de Brown et Famiglietti ne feraient qu'ajouter un chapitre préliminaire à l'analyse de Sarah Hanley, si les différentes fonctions du lit ne s'entremêlaient constamment. En effet, d'un côté, cette dimension justicière ne cesse d'importer jusqu'à la fin de l'Ancien Régime, comme l'a montré David Parker ${ }^{9}$; de l'autre, les lits de justice, ou leurs équivalents innommés du xIv siècle, portent aussi sur des matières qui relèvent du prétendu « droit public » et notamment sur la question de la majorité du roi. L'ordonnance cabochienne de 1413 et son abolition font aussi l'objet de lits de justice. En outre, l'utilisation du lit de justice pour imposer l'enregistrement d'édits apparaît dès $1563^{10}$; dès lors, les protestations du Parlement, que Sarah Hanley voient apparaître à la fin du xvir siècle, dans sa troisième phase, " absolutiste ", du lit de justice, se manifestent un bon siècle auparavant.

Une radicale opposition historiographique, entre discontinuisme et continuisme, distingue les deux ouvrages. Pour Sarah Hanley, le lit de justice ne peut prendre qu'un sens à la fois, par une succession de ruptures. Pour Brown et Famiglietti, les sens du lit de justice s'élaborent ensemble dans une longue durée que le contexte spécifie de temps à autre. Cette orientation de Sarah Hanley tient à l'influence de deux disciplines : l'histoire intellectuelle, telle qu'elle fut léguée par Ernst Kantorowicz à Ralph Giesey, privilégie la vie propre d'un concept, en le dégageant précisément des contextes qui en entravent la perception. Dès lors, il faut donner à cette grêle entité le tuteur rigide de catégorisations fortes (comme la regrettable notion d'absolutisme). Ce danger qui guettait l'œuvre de Kantorowicz sans l'envahir, tant cet historien savait jouer sur des déplacements de sources et d'objets, se fait plus menaçant lorsqu'on s'attache à des objets à la fois limités et concrets comme les cérémonies politiques. Par ailleurs, le moment central de la démonstration de Sarah Hanley se situe dans la phase "constitutionnelle", au début du xvi siècle; il importe d'affirmer que le politique se construit d'abord par le rite, juste avant les grandes élaborations discursives de la fin du siècle, comme s'il fallait justifier cette certitude de l'anthropologie culturelle : l'authenticité et l'origine se trouvent du côté

9. Voir David PARKER, «Sovereignty, absolutism and the function of the law in 17 th century France ", Past and Present, 122, 1989, p. 36-74.

10. Voir M. P. Holt, art. cit. supra n. 1. 
du rite. Il fallait donc constituer le lit de justice en « texte », selon les injonctions de Clifford Geertz, qui, en traitant ainsi les pratiques et les objets culturels, les dotent d'une fallacieuse autonomie" ${ }^{11}$ Et malgré la polysémie que pourrait suggérer la notion de «texte ", l'anthropologie culturelle tend à réduire les processus à des conduites symboliques de sens univoque, sans doute parce que le véritable auteur du " texte » n'est pas l'acteur de l'histoire, mais l'auteur déchiffreur.

Or les cérémonies politiques, malgré leurs charges d'effets symboliques, sont aussi des actes et des processus concrets, susceptibles d'appropriations, de détournements divers. C'est ce que j'ai essayé de montrer à propos des funérailles de Charles VIII, en déployant des interprétations contextuelles qui pouvaient se juxtaposer à l'interprétation constitutionnelle et univoque de Ralph Giesey ${ }^{12}$. Dans le cas du lit de justice, Brown et Famiglietti suggèrent qu'une étude précise de l'interaction politique entre le parlement de Paris et la monarchie pourrait compliquer utilement l'histoire du lit de justice. En effet, un tournant essentiel de l'histoire de l'institution se situe en 1485 , quand une ordonnance de Charles VIII proclame que le lit de justice doit se tenir en son parlement de Paris et nulle part ailleurs. Cela n'implique pas une localisation géographique, mais institutionnelle : le Parlement peut être convoqué hors de Paris. En cette fin de $\mathrm{xv}^{e}$ siècle, où Charles VIII et Louis XII ont besoin de diversifier leurs réseaux de soutien, la formulation royale entend honorer et flatter le Parlement, qui est régulièrement comparé au Sénat romain. Dès lors, le Parlement peut prétendre être à la source virtuelle ou actuelle du droit et de la loi. C'est dire que l'histoire du lit de justice doit se comprendre au sein des rapports de pouvoir qui s'élaborent autour du parlement de Paris, dans la variété successive de sa composition. Cette interaction rend compte aussi des incohérences, des lacunes et des sauts qui semblaient justifier le discontinuisme de Sarah Hanley : dans la seconde moitié du $\mathrm{xvI}^{\mathrm{e}}$ siècle, de nombreuses séances royales du Parlement ne reçoivent pas le nom de lit de justice; Jean Du Tillet, au cours des quarante ans de sa carrière, change les critères de définition du lit de justice. C'est que l'interaction s'est transformée en une sourde lutte : là où les monarques entendent multiplier la forme cérémonielle, notamment en faisant un lit de justice de toute séance royale succédant à une entrée, le Parlement refuse de faire noter dans les archives que telle séance est un lit de justice, puisque désormais, le Parlement se considère lui-même comme lit de justice. La position compliquée de Jean Du Tillet, admirablement étudiée par Elizabeth Brown dans une série d'articles, parus avant et après l'ouvrage sur le lit de justice ${ }^{13}$, rend compte des oscillations de sa théorie et de sa pratique; témoin et greffier, il est également un grand archiviste. Rival de son frère Séraphin qu'il supplante en 1530 contre le vœu du Parlement, il doit beaucoup au roi tout en tirant sa gloire et sa carrière du Parlement. C'est dire que le principal

11. Voir Giovanni LevI, "I pericoli del geertzismo ", Quaderni storici, 58, 1985, p. 269 277 et Pierre Bourdreu, Méditations pascaliennes, Paris, Seuil, 1997, p. 66-67.

12. A. Bouread, Le Simple Corps du roi. L'impossible sacralité des souverains français, $x v^{e}-\mathrm{xvm}{ }^{e}$ siècle, Paris, Éditions de Paris, 1988.

13. E. Brown, « Le greffe civil du parlement de Paris au xvi siècle. Jean Du Tillet et les registres des plaidoiries ", Bibliothèque de l'École des chartes, t. 153, 1995, p. 327-372. Ead., "Jean Du Tillet, François $1^{\text {er }}$ and the Trésor des chartes", in Histoires d'archives. Recueil d'articles offert à Lucie Fossier par ses collègues et amis, Paris, Société des amis des Archives de France, 1997, p. 237-247. 
témoin et théoricien du lit de justice, loin d'être un scribe flagorneur, participe à l'interaction où se jouent la réalité et l'image du lit de justice.

Il est probable que l'opposition entre les deux grands ouvrages sur le lit de justice a été un peu forcée et qu'il faille désormais tenter de combiner les deux études, d'autant que, pour le $\mathrm{xvIl}^{\mathrm{e}}$ et le xvIII siècle, la méthode de Sarah Hanley produit moins de distorsions, puisque le lit de justice a réellement acquis l'autonomie d'existence que lui ont conférée les combats textuels et politiques du xvi siècle et les transformations du rôle du parlement de Paris. Mais, au-delà d'incontestables progrès dans la connaissance de l'institution cérémonielle, la différence de résultats illustre les possibilités qu'offre une histoire soucieuse d'interactions, de changements d'échelle et de contexte. Le débat aidera à penser les conditions pour une nouvelle histoire de l'institution ${ }^{14}$ qui saura enfin conjoindre l'autonomie de l'objet et son historicité.

$$
\begin{array}{r}
\text { Alain BoUREAU, } \\
\text { École des hautes études en sciences sociales, } \\
54, \text { bd Raspail, } \\
75006 \text { Paris } \\
\text { (juillet 1997). }
\end{array}
$$

14. Voir Jacques REVEL, "L'institution et le social ", in Les Formes de l'expérience. Une autre histoire sociale, dir. Bernard LEPETIT, Paris, Albin Michel («L'Évolution de l'humanité »), 1995, p. 63-84. 